\title{
Francolebias arvernensis n. sp., une nouvelle espèce de poissons cyprinodontiformes oligocènes de Chadrat (Saint- Saturnin, Puy-de-Dôme, France), avec une brève notice sur un Umbridae fossile du même gisement
}

\author{
Jean GAUDANT+ \\ Centre de Recherche sur la Paléobiodiversité et les Paléoenvironnements, \\ USM 203, UMR 7207 (MNHN, CNRS, UPMC, Sorbonne Universités), \\ Muséum national d'histoire naturelle, Département Histoire de la Terre \\ case postale 38, 57 rue Cuvier, F-75231 Paris cedex 05 (France)
}

Publié le 30 septembre 2016

MOTS CLÉS Cyprinodontiformes,

Valenciidae,

Umbridae,

Oligocene

Puy-de-Dôme,

paléoécologie, espèce nouvelle.

KEY WORDS Cyprinodontiforms,

Valenciidae,

Umbridae,

Oligocene,

Central France,

palaeoecology,

new species.
urn:Isid:zoobank.org:pub:79306470-A198-4043-8AAF-D3DE16E09427

Gaudant $†$ J. 2016. - Francolebias arvernensis n. sp., une nouvelle espèce de poissons cyprinodontiformes oligocènes de Chadrat (Saint-Saturnin, Puy-de-Dôme, France), avec une brève notice sur un Umbridae fossile du même gisement. Geodiversitas 38 (3): 435-449. http://dx.doi.org/10.5252/g2016n3a4

\section{RÉSUMÉ}

L'étude anatomique de nouveaux matériaux récoltés dans les sapropels oligocènes de Chadrat (SaintSaturnin, Puy de Dôme, France) permet de préciser les caractères de ces poissons cyprinodontiformes. La comparaison avec la description originale de poissons du sapropel de Chadrat publiée par Piton (1935) conduit à mettre en évidence des différences significatives entre cette description et nos propres observations. En raison de l'absence de figuration originale de l'espèce Haplochilus piacentini aggravée par la perte de son holotype, le nom Haplochilus piacentini doit être considéré comme un nomen nudum. La présente étude montre que les poissons fossiles de Chadrat appartiennent à une nouvelle espèce du genre Francolebias Costa, 2012: F arvernensis n. sp. On signale également la présence dans ce gisement d'un squelette d'un Umbridae primitif: Palaeoesox cf. weileri (Martini, 1965).

\section{ABSTRACT}

Francolebias arvernensis $n$. sp., a new species of Oligocene cyprinodontiform fishes from Chadrat (SaintSaturnin, Puy-de-Dôme, France), with a short notice on a fossil umbrid from the same locality.

The anatomical study of new materials collected in the Oligocene paper shales of Chadrat (SaintSaturnin, Puy-de-Dôme, France) allows a precise description of these cyprinodontiform fishes. The comparison with the original description of fossil fishes from the Chadrat sapropel published by Piton (1935) leads one to emphasize significant differences between this description and our own observations. Because of the lack of original figuration of the species Haplochilus piacentini, and of the loss of its holotype, the name Haplochilus piacentini should be considered as a nomen nudum. The present study has shown that the fossil fishes from Chadrat belong to a new species of the genus Francolebias Costa, 2012: F. arvernensis n. sp. Additionally, the occurrence in this locality of the skeleton of a primitive umbrid: Palaeoesox cf. weileri (Martini, 1965) is reported. 


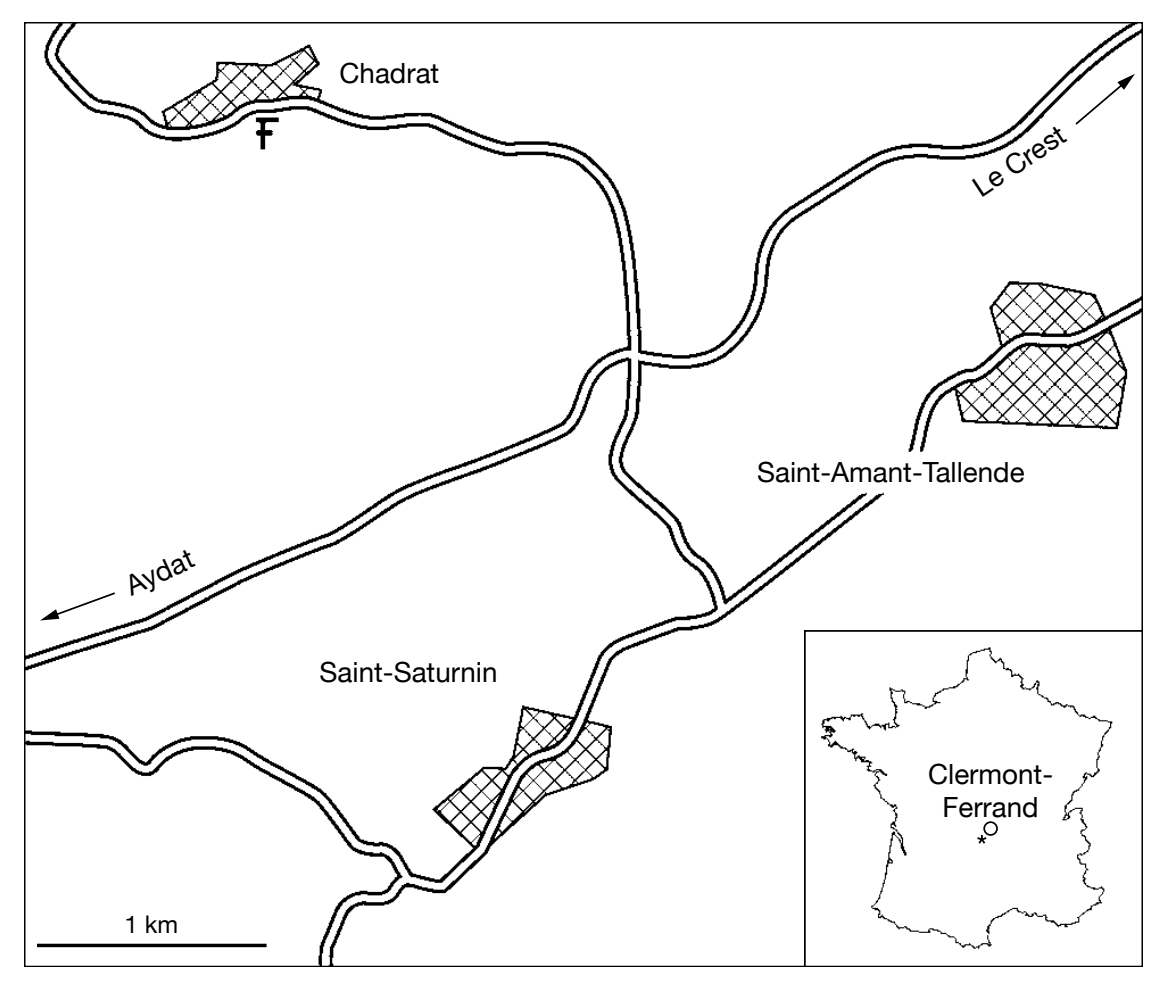

FIG. 1. - Carte de localisation du gisement de Chadrat, près de Saint-Saturnin (Puy-de-Dôme, France).

\section{INTRODUCTION}

Dans une brève notice, Piton (1935) décrivit les poissons fossiles découverts par le Père René Piacentini dans les schistes carton (dussodyle) qui affleurent sur la rive droite du Taux, sous quelques décimètres de calcaire en plaquettes, près du village de Chadrat (commune de Saint-Saturnin, Puy-deDôme, France) (Fig. 1).

Piton crut devoir attribuer ces fossiles à l'espèce Prolebias stenoura (Sauvage, 1874), à l'exception d'un seul spécimen pour lequel il créa l'espèce Haplochilus piacentini. Aucune figure n'accompagnait la description de cette espèce alors qu'au Second Congrès géologique international, qui s'est tenu à Bologne en 1881, il avait été décidé qu' «À l'avenir, pour les noms spécifiques, la priorité ne sera irrévocablement acquise que lorsque l'espèce aura été non seulement décrite, mais figurée». Cette espèce doit donc être considérée comme un nomen nudum, cela d'autant plus que l'holotype de cette espèce semble être resté la propriété du Père Piacentini, qui enseignait à l'École des missions des Frères du Saint-Esprit de Cellule (Puy-de-Dôme). Or, au cours des années 1970, la fermeture de cette école entraîna le transfert de la collection du Père Piacentini au siège de sa Congrégation, où ce fossile n’a pas été retrouvé. De ce fait, le seul élément qui permette de se faire une idée de l'espèce Haplochilus piacentini est la description publiée par cet auteur. En réalité, l'étude à laquelle nous nous sommes livré nous a convaincu que les Cyprinodontiformes de Chadrat appartiennent à une seule espèce qui diffère de l'espèce Prolebias stenoura, du gisement de Corent-Sainte-Marguerite.

\section{MATÉRIEL ÉTUDIÉ}

Le matériel étudié dans le présent article a été principalement recueilli par nos soins en juillet 1976 et juillet 1977 dans le dussodyle de Chadrat. Un spécimen provient en outre de calcaires en plaquettes sus-jacents. Ce matériel, ainsi que les spécimens généreusement offerts par M. Philippe Olivier, est conservé à Paris dans les collections paléontologiques du Muséum national d'Histoire naturelle sous les numéros de catalogue MNHN.F.CHD1 à 61. S'y ajoute le squelette d'un Umbridae recueilli dans le calcaire en plaquettes (MNHN.F.CHD62).

\section{SYSTÉMATIQUE}

Famille ValenciIdae Parenti, 1981

Genre Francolebias Costa, 2012

Francolebias arvernensis $\mathrm{n}$. $\mathrm{sp}$.

(Figs 2-18)

HolotYPE. — Spécimen MNHN.F.CHD1.

GISEMENT TYPE. - Sapropels et calcaires affleurant en rive droite du Taux entre le village et le cimetière de Chadrat.

ÂGE. — Oligocène basal.

Origine Du nOM. — De Arvernis, l'Auvergne. 


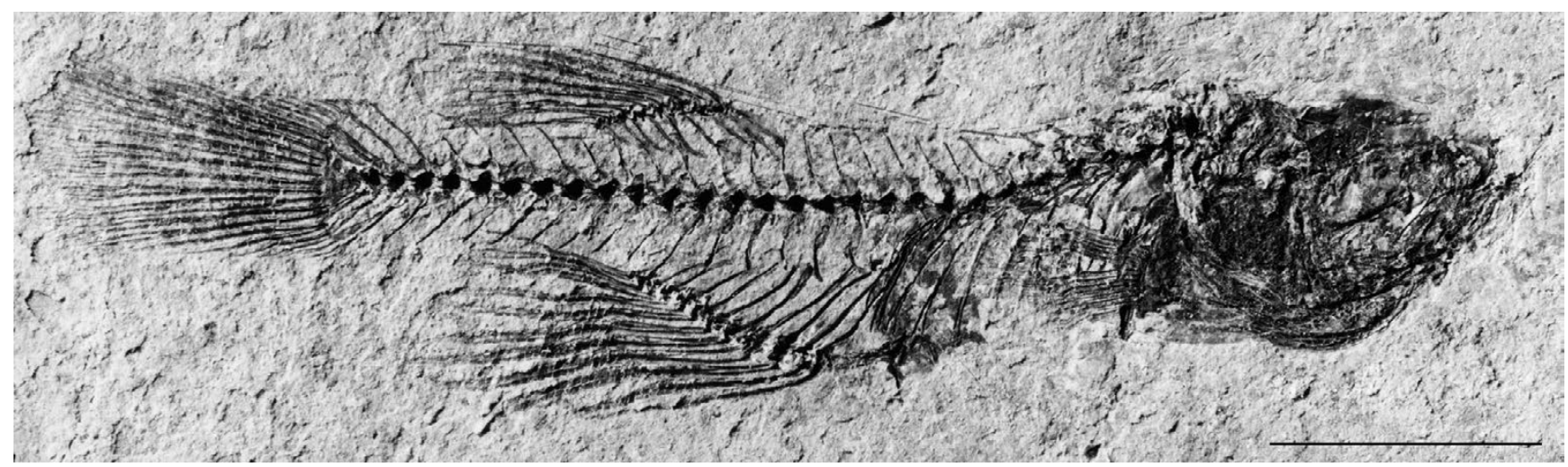

FIG. 2. - Francolebias arvernensis n. sp., vue générale de l'holotype conservé à Paris, dans les collections paléontologiques du Muséum national d'Histoire naturelle sous le numéro MNHN.F.CHD1G. Échelle: $5 \mathrm{~mm}$.

TABLEAU 1. - Mensurations (en mm) de trois spécimens de Francolebias arvernensis $\mathrm{n}$. sp. Entre parenthèses est indiqué le pourcentage de la longueur standard.

\begin{tabular}{|c|c|c|c|c|c|c|}
\hline \multirow{2}{*}{$\frac{\text { Mensurations }}{\text { Longueur totale }}$} & \multicolumn{2}{|c|}{ MNHN.F.CHD1 (holotype) } & \multicolumn{2}{|c|}{ MNHN.F.CHD31 } & \multicolumn{2}{|c|}{ MNHN.F.CHD15 } \\
\hline & 26,5 & - & 26,5 & - & 28,5 & - \\
\hline Longueur standard & 21,5 & - & 21,5 & - & 22,5 & - \\
\hline Hauteur maximale du corps & 4,5 & $(20,9 \%)$ & 3,5 & $(16,2 \%)$ & 4 & $(17,7 \%)$ \\
\hline Longueur de la tête & 6 & $(27,9 \%)$ & 6,5 & $(30,2 \%)$ & 6 & $(26,6 \%)$ \\
\hline Distance antédorsale & 14 & $(65,1 \%)$ & 14,5 & $(67,4 \%)$ & 13,5 & $(60 \%)$ \\
\hline Distance antéanale & 12,5 & $(58,1 \%)$ & 13,5 & $(62,7 \%)$ & 13 & $(57,7 \%)$ \\
\hline Distance antépectorale & 6,5 & $(30,2 \%)$ & 7 & $(32,5 \%)$ & 7 & $(31,1 \%)$ \\
\hline Distance antépelvienne & 11 & $(51,1 \%)$ & 11,5 & $(53,4 \%)$ & 11,5 & $(51,1 \%)$ \\
\hline Longueur de la dorsale & 4,5 & $(20,9 \%)$ & c. 3,5 & $(16,2 \%)$ & 5 & $(22,2 \%)$ \\
\hline Longueur de l'anale & 6 & $(27,9 \%)$ & 4,5 & $(20,9 \%)$ & 7 & $(31,1 \%)$ \\
\hline Longueur des pectorales & 3 & $(13,9 \%)$ & - & - & 3,5 & $(15,5 \%)$ \\
\hline Longueur des pelviennes & 1,5 & $(6,9 \%)$ & 1,5 & $(6,9 \%)$ & 1,5 & $(6,6 \%)$ \\
\hline Longueur basale de la dorsale & 2,5 & $(11,6 \%)$ & 2 & $(9,3 \%)$ & 3 & $(13,3 \%)$ \\
\hline Longueur basale de l'anale & 4 & $(18,6 \%)$ & 2,5 & $(11,6 \%)$ & 4 & $(17,7 \%)$ \\
\hline Longueur du pédicule caudal & 5 & $(23,2 \%)$ & 5 & $(23,2 \%)$ & 5 & $(22,2 \%)$ \\
\hline Hauteur du pédicule caudal & 3 & $(13,9 \%)$ & 2,5 & $(11,6 \%)$ & 3 & $(13,3 \%)$ \\
\hline
\end{tabular}

DiAGNOSE. - Petits poissons cyprinodontiformes dont la longueur standard ne semble pas avoir excédé $30 \mathrm{~mm}$. Corps élancé. Tête massive dont la longueur excède légèrement le quart de la longueur standard. Bouche oblique dont les mâchoires portent une seule rangée de petites dents coniques. Colonne vertébrale comportant 30 ou 31 vertèbres, dont 18-19 postabdominales. Côtes pleurales longues et robustes au nombre d'environ neuf paires. Nageoire caudale en forme de palette faiblement arrondie à son extrémité, composée de 10 à 12 rayons à la fois articulés et bifurqués et de 8 ou 9 rayons marginaux dorsaux et ventraux. Dorsale assez longue débutant nettement en arrière de la verticale passant par l'origine de l'anale; $\mathrm{i}-\mathrm{ii}+\mathrm{I}+7-8$ rayons supportés par $9(10$ ?) ptérygiophores. Anale grande, assez longue: i-ii+ I+ (8) 9-11 rayons, soutenue par 10-12 ptérygiophores. Pectorales de taille médiocre; $14-15$ rayons. Pelviennes très petites insérées à faible distance de l'anale; 6 rayons. Os pelviens courts et massifs. Écailles cycloïdes assez grandes.

\section{DESCRIPTION ANATOMIQUE}

Les Cyprinodontiformes des schistes carton de Chadrat sont de petits poissons au corps élancé dont la longueur standard ne semble pas avoir excédé $30 \mathrm{~mm}$, cette dimension étant généralement comprise entre 20 et $25 \mathrm{~mm}$. La hauteur maximale du corps, mesurée à l'avant de la nageoire anale, est comprise environ 5 à 6 fois dans la longueur standard (Fig. 2).

La tête, massive, est grande: sa longueur excède légèrement le quart de la longueur standard. La nageoire caudale,

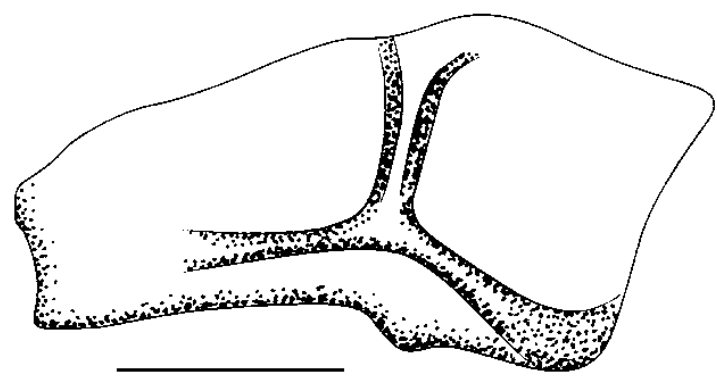

FIG. 3. - Francolebias arvernensis n. sp., frontal isolé fossilisé sur la pièce MNHN.F.CHD2B. Échelle: $1 \mathrm{~mm}$.

en forme de palette légèrement arrondie à son extrémité distale, mesure environ un quart de la longueur standard. La nageoire dorsale qui débute sensiblement en arrière de la verticale passant par l'origine de l'anale, débute approximativement aux $2 / 3$ de la longueur du corps, mesurée de la pointe $\mathrm{du}$ museau au bord postérieur des hypuraux. Les nageoires pelviennes sont insérées sensiblement plus près de l'origine de l'anale que de la base des pectorales.

Les mensurations de trois spécimens ont été regroupées dans le Tableau 1. 


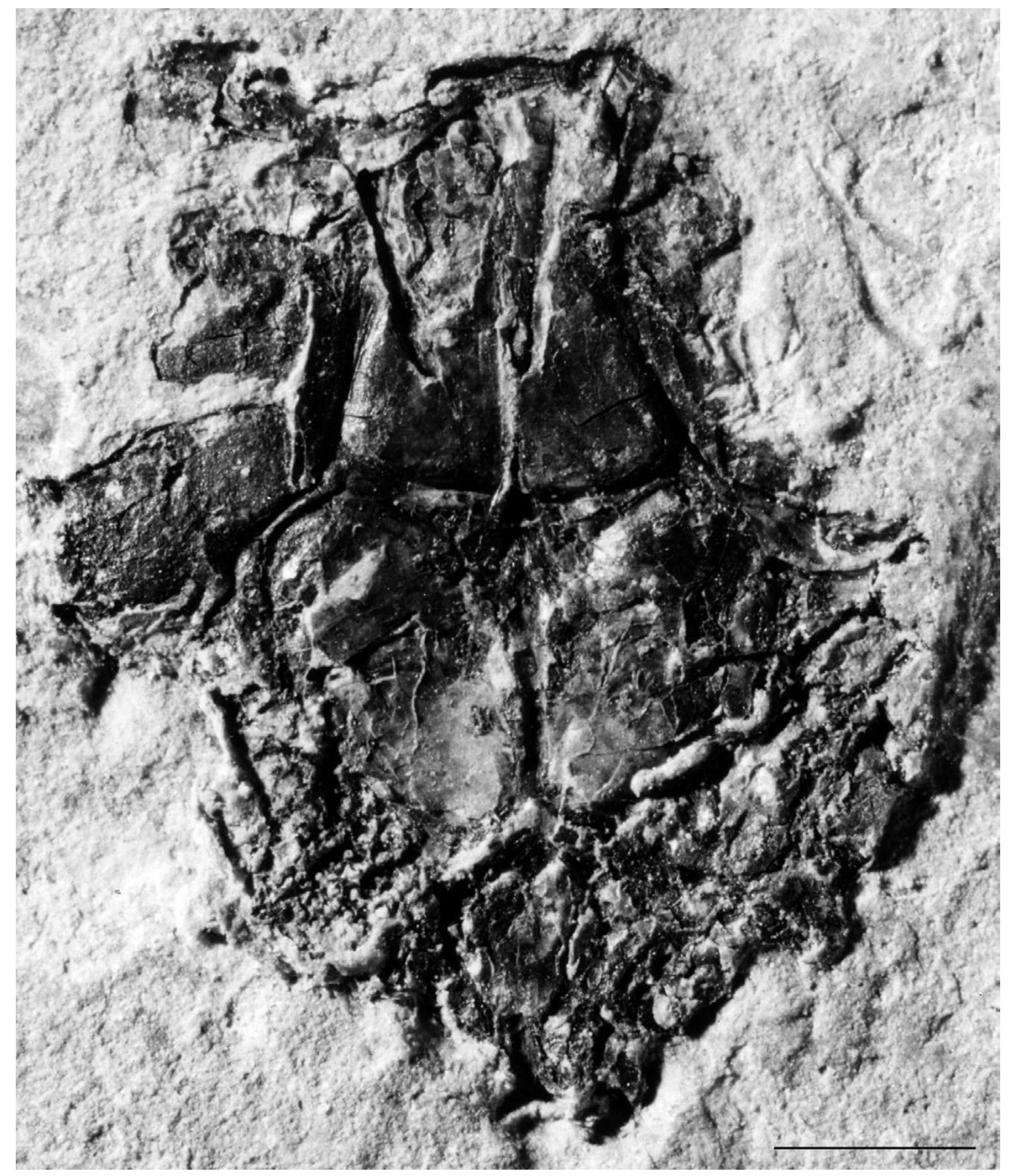

FIG. 4. - Francolebias arvernensis n. sp., toit crânien fossilisé sur la pièce MNHN.F.CHD3. Échelle: 1 mm.

La tête

La récolte d'un certain nombre d'os isolés a permis de pallier la qualité de conservation relativement médiocre des squelettes qui sont fossilisés sous forme d'empreintes.

Orbite. Elle est grande car son diamètre horizontal égale plus du tiers de la longueur de la tête. Elle est traversée approximativement en son milieu par le parasphénoïde, qui s'abaisse légèrement vers l'arrière. La série infraorbitaire est réduite au seul lacrymal, d'assez grande taille, dont l'extrémité postérieure effilée atteint approximativement le milieu du bord inférieur de l'orbite. La bouche, très oblique, est courte, l'articulation de la mandibule avec le crâne étant située un peu en avant de la verticale passant par le bord antérieur de l'orbite.

Frontaux (Fig. 3). Les frontaux (Fr) sont larges, notamment dans leur région postorbitaire, comme le montre un os fossilisé sur la pièce MNHN.F.CHD2B. À proximité du bord latéral 


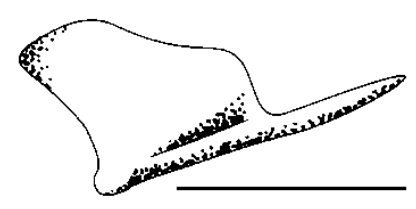

FIG. 5. - Francolebias arvernensis n. sp., carré isolé fossilisé sur la pièce MNHN.F.CHD4. Échelle: 1 mm.

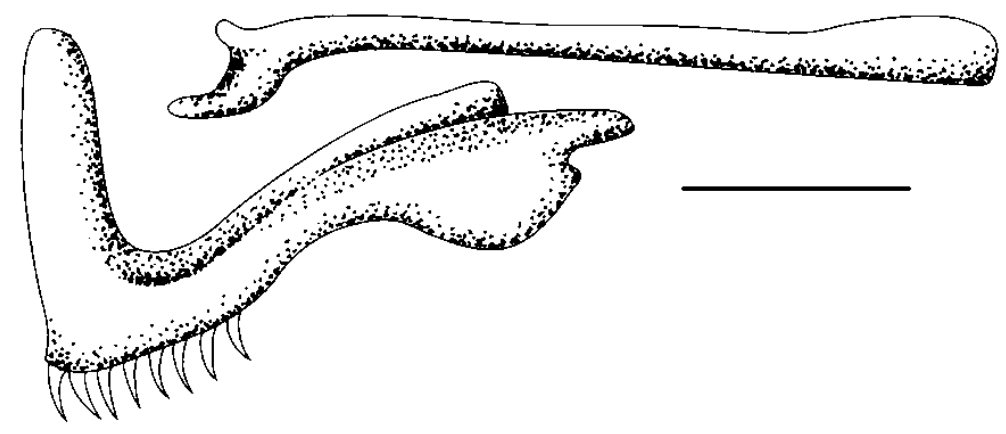

FIG. 6. - Francolebias arvernensis n. sp., prémaxillaire et maxillaire isolés fossilisés sur la pièce MNHN.F.CHD2B. Échelle: 1 mm.

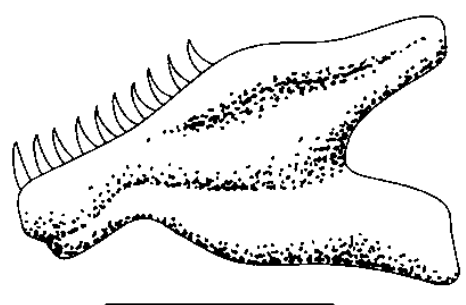

FIG. 7. - Francolebias arvernensis n. sp., dentaire isolé fossilisé sur la pièce MNHN.F.CHD2A. Échelle: 1 mm.

de l'os s'observe le tracé du canal supraorbitaire (c.s.o.). Un peu en avant du tiers postérieur de l'os, ce canal émet deux diverticules (vérif.) disposés plus ou moins parallèlement en direction du plan sagittal où le canalicule antérieur paraît entrer en contact avec son symétrique, comme le montre le toit crânien fossilisé sur la pièce MNHN.F.CHD3 (Fig. 4). Ce toit crânien permet d'observer la présence d'un grand supraoccipital (Socc) dont la partie antérieure effilée vers l'avant s'immisce entre les frontaux. Ce toit crânien permet également de constater l'absence des pariétaux.

Région ptérygo-carrée. Elle est partiellement observable. Le carré, qui a été observé à l'état isolé sur la pièce MNHN.F.CHD4 (Fig. 5), a une forme triangulaire. Il présente une profonde échancrure qui entaille sa partie postérieure, délimitant en arrière un long processus qui se termine par une extrémité pointue. Son processus articulaire est légèrement déjeté vers l'avant. Le symplectique, fossilisé sur le spécimen MNHN.F.CHD8D, CHD8G, est long et robuste. Son extrémité distale s'engage profondément dans la gouttière articulaire du carré.

Prémaxillaire. Il a été observé à l'état isolé sur la pièce MNHN.F.CHD2B, de même que le maxillaire (Fig. 6). Il est caractérisé par le grand développement de son processus ascendant qui se termine distalement par une extrémité arrondie. Le processus alvéolaire du prémaxillaire, faiblement concave, se termine postérieurement par une région renflée effilée vers l'arrière. Sa partie antérieure porte une rangée de petites dents coniques légèrement arquées. Le maxillaire, fossilisé à l'état isolé sur la pièce MNHN.F.CHD2B, est un os en forme de baguette allongée très étroite, qui se termine vers l'avant par un processus articulaire permettant les mouvements du prémaxillaire.

Mandibule. Très courte, elle est aussi relativement massive. Sa longueur égale à peine le tiers de la longueur de la tête. Le dentaire a été observé à l'état isolé sur la pièce MNHN.F.CHD2A (Fig. 7). Sa hauteur maximale égale près des $2 / 3$ de sa longueur. Son bord oral faiblement convexe, dont la moitié antérieure porte une série de petites dents coniques. Son bord ventral est, au contraire, faiblement concave. La région symphysaire est recourbée vers le bas. L'arrière de l'os est fortement échancré pour recevoir la partie antérieure de l'angulaire.

Préopercule. Il est conservé à l'état isolé. Celui qui est fossilisé sur la pièce cataloguée MNHN.F.CHD5 (Fig. 8) montre le grand développement de la branche horizontale, qui est plus longue que la branche verticale à laquelle elle se raccorde postérieurement par un contour arrondi. Les deux branches du canal préoperculaire déterminent un angle droit. En avant de cet angle prend place une lamelle osseuse subtriangulaire. 


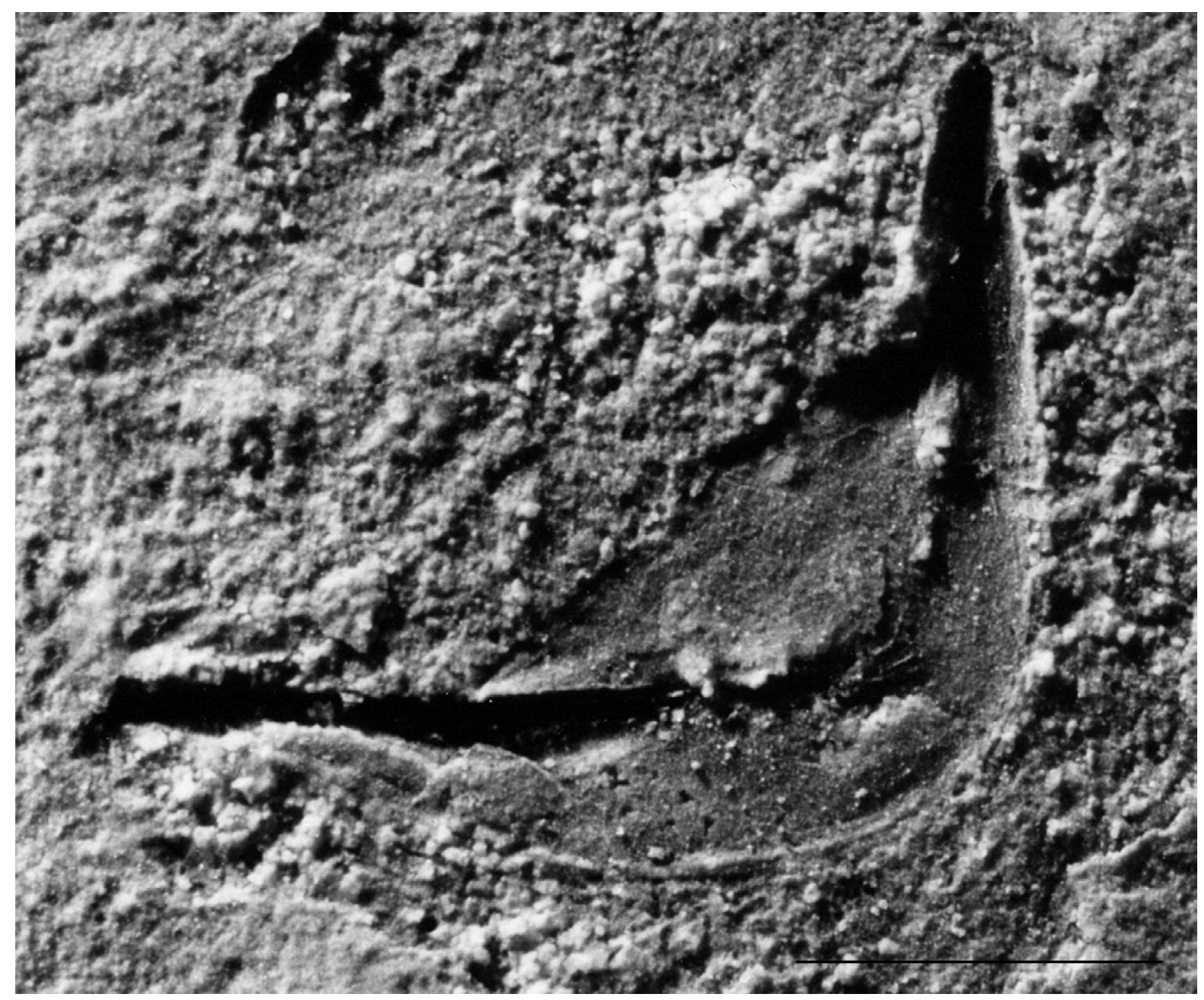

FIG. 8. - Francolebias arvernensis n. sp., préopercule isolé fossilisé sur la pièce MNHN.F.CHD5. Échelle: 1 mm.

Opercule. Il est, sans aucun doute, l'os qui a été fossilisé le plus fréquemment à l'état isolé. De forme générale triangulaire, il est caractérisé par son bord dorsal faiblement concave, comme le montre la pièce MNHN.F.CHD6 (Fig. 9). Sa largeur maximale égale environ les $3 / 4$ de sa hauteur.

Sousopercule. Il est observable à l'état isolé sur la pièce MNHN.F.CHD7A. C'est un os crescentiforme relativement grand dont les bords antérieur et postérieur se raccordent ventralement par une courbe régulière (Fig. 10). Son bord supérieur est concave. À sa partie antérieure prend place le processus assurant l'articulation avec l'opercule.

Arc hyoïde. Il est partiellement observable. L'hyomandibulaire est fossilisé à l'état isolé sur la pièce MNHN.F.CHD2B, où l'on observe sa face mésiale (Fig. 11). Sa tête articulaire triangulaire est perforée par le foramen qui permet le passage du tronc hyomandibulaire du nerf facial. Le processus opercularis est court et relativement large.

Le cératohyal distal est fossilisé à l'état isolé sur la pièce MNHN.F.CHD2B (Fig. 12). Cet os présente une constriction bien marquée dans sa partie médiane, ce qui lui confere une forme en clepsydre. Toutefois, sa région postérieure est beaucoup plus développée que sa partie antérieure. Six rayons branchiostèges acinaciformes s'articulent avec les cératohyaux proximal et distal, comme le montre le spécimen MNHN.F.CHD8G (Fig. 13).

\section{Le corps}

Colonne vertébrale. Elle se compose d'une trentaine de vertèbres (30 ou 31). On dénombre le plus souvent 18 ou 19 vertèbres postabdominales et, exceptionnellement, seulement 17. Dans la région postabdominale, les centra sont sensiblement plus longs que hauts. Ils portent des neurapophyses et des hémapophyses très développées qui atteignent au moins les $3 / 4$ de la distance séparant les bords dorsal et ventral des centra respectivement des bords dorsal et ventral de la région postabdominale du corps.

Côtes pleurales. Longues et robustes, elles semblent avoir été au nombre de neuf paires. Sessiles, leur extrémité distale atteint pratiquement le bord ventral de la cavité abdominale.

Epineuralia. Relativement grêles, au nombre d'au moins sept ou huit, elles sont observables dans la région abdominale du spécimen MNHN.F.CHD35. 


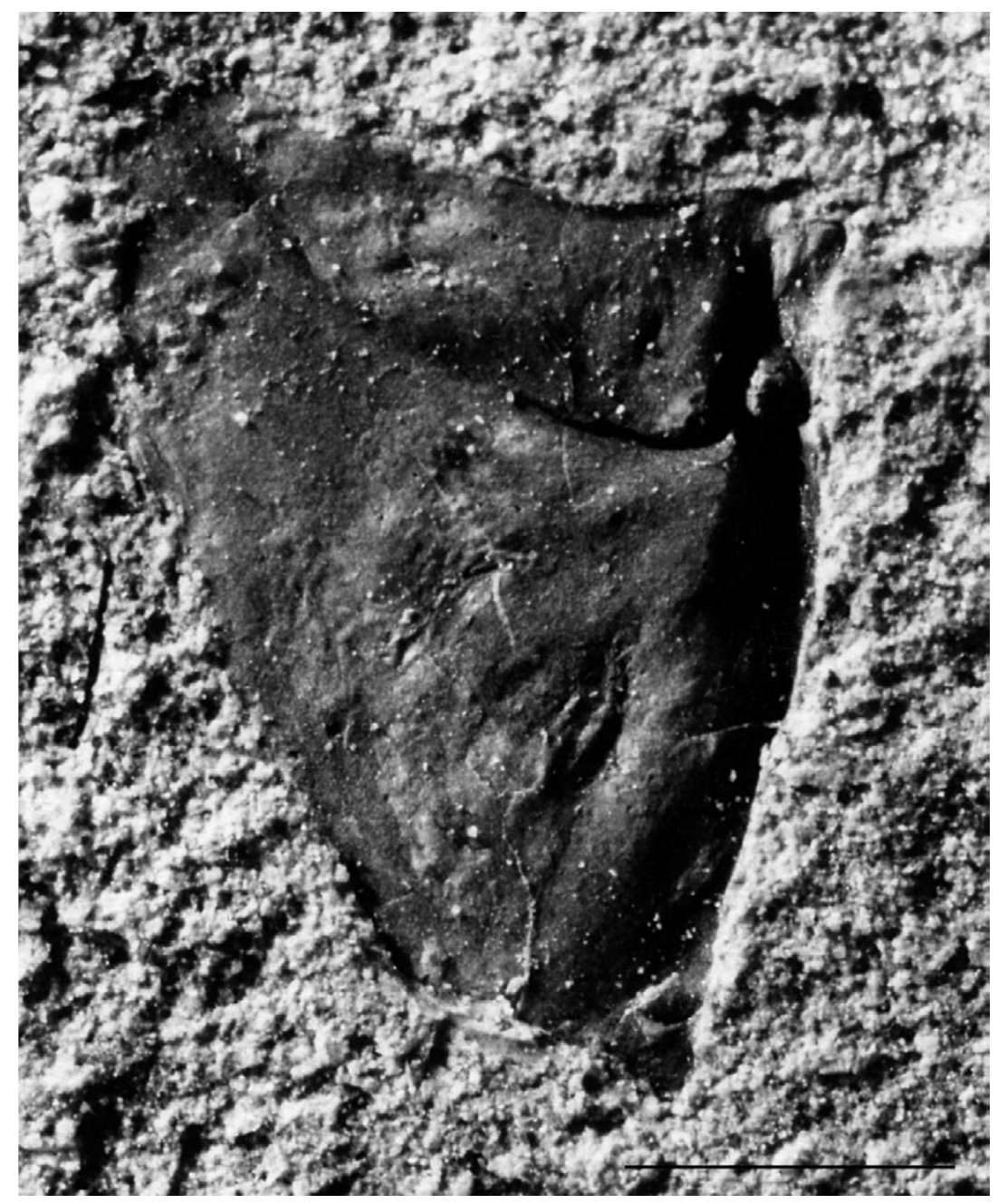

FIG. 9. - Francolebias arvernensis n. sp., opercule isolé fossilisé sur la pièce MNHN.F.CHD6. Échelle: $1 \mathrm{~mm}$.

Nageoire caudale. La nageoire caudale, en forme de palette légèrement convexe à son extrémité distale, se compose de 28 rayons dont une douzaine sont à la fois articulés et bifurqués (toutefois, nous n'en avons dénombré que 10 sur le spécimen MNHN.F.CHD17). Ces rayons principaux sont précédés, dorsalement et ventralement, par 8 ou 9 rayons marginaux dont seuls les plus longs sont articulés distalement.

La nageoire caudale est soutenue par un endosquelette constitué de quatre centra dont les neurapophyses et hémapophyses allongées et plus ou moins modifiées concourent au soutien des rayons de la caudale (Fig. 14A, B). Comme le montre le spécimen MNHN.F.CHD1G, il se compose d'un complexe uro-terminal (PU1+U1) que précèdent trois centra préuraux libres (PU2 à PU4). Le complexe uro-terminal supporte une plaque hypurale unique très large $(\mathrm{Hy})$ dont la longueur du bord postérieur excède celle de ses bords inférieur et supérieur. Ventralement, prend place le parhypural (PHy), relativement étroit qui s'articule avec la partie ventrale du complexe uro-terminal. Dorsalement, s'observe un épural unique (Ep) dont la région proximale est élargie. Il occupe une position pratiquement symétrique de celle du parhypural. En avant de l'épural prend place une neurapophyse dédoublée (a.n. PU2a et p) très longue, portée par le centrum préural libre postérieur. Ventralement, l'hémapophyse portée par ce même centrum (a.h. PU2) est à la fois très longue et aplatie dans le plan sagittal, comme l'est également la partie distale de l'hémapophyse précédente (a.h. PU3). Sur plusieurs spécimens, s'observe le dédoublement des neurapophyses et des hémapophyses portée par les deux centra préuraux libres postérieurs (PU2 et PU3).

La nageoire dorsale, de taille modérée, occupe une position postérieure: elle débute en effet approximativement au-dessus du milieu de la base de la nageoire anale. La distance antédorsale égale environ $65 \%$ de la longueur du corps mesurée de la pointe du museau au bord postérieur des hypuraux. La nageoire dorsale se compose généralement de 10 ou 11 rayons, dont 7 ou 8 (plus rarement 9) sont à la fois articulés et bifurqués. La longueur de ceux-ci diminue assez rapidement vers l'arrière. De ce fait, la nageoire dorsale paraît tronquée postérieurement. À l'avant de la nageoire on dénombre deux ou trois rayons non bifurqués dont la longueur s'accroît d'avant en arrière. La longueur du dernier d'entre eux, qui est le plus long de la nageoire, égale environ une fois et demie la hauteur maximale du corps.

L' endosquelette de la nageoire dorsale est composé de 9 ou 10 ptérygiophores dont la longueur diminue légèrement vers l'arrière à partir du troisième. 


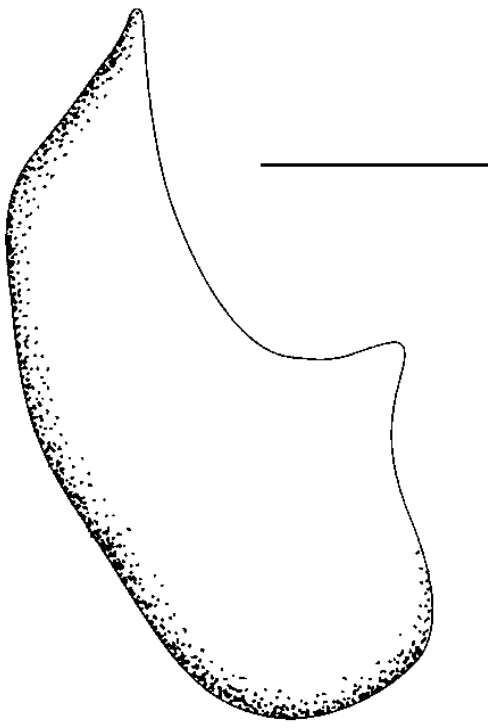

FIG. 10. - Francolebias arvernensis n. sp., sousopercule isolé fossilisé sur la pièce MNHN.F.CHD7A. Échelle: $1 \mathrm{~mm}$.

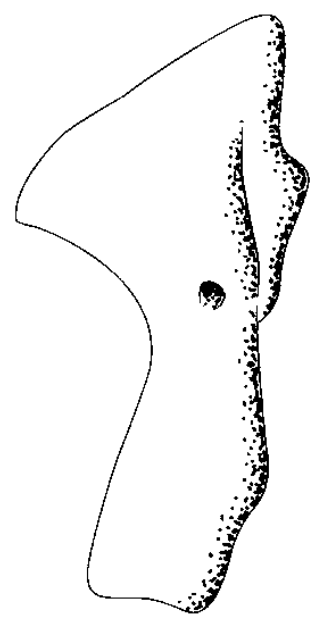

FIG. 11. - Francolebias arvernensis n. sp., hyomandibulaire isolé. Face mésiale. Os fossilisé sur la pièce MNHN.F.CHD2B. Échelle: $1 \mathrm{~mm}$.

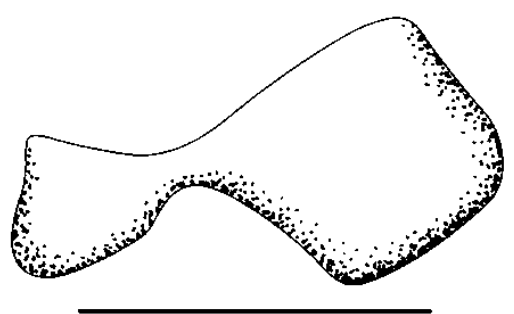

FIG. 12. - Francolebias arvernensis n. sp., cératohyal distal isolé fossilisé sur la pièce MNHN.F.CHD2B. Échelle: $1 \mathrm{~mm}$.

Nageoire anale. La nageoire anale, très grande, occupe une position très avancée par rapport à la dorsale. La distance antéanale égale en effet environ $60 \%$ de la longueur du corps. De ce fait, seule sa moitié postérieure est opposée à la dorsale. Elle se compose de 11 à 13 rayons, dont 9 à 11 (plus rarement 8) sont à la fois articulés et bifurqués. Comme c’est le cas pour la nageoire dorsale, la longueur de ces rayons diminue assez rapidement vers l'arrière, ce qui donne à cette nageoire une forme tronquée verticalement à l'arrière. À l'avant de la nageoire prennent place deux ou trois rayons articulés non bifurqués. Le dernier d'entre eux est le plus long rayon de la nageoire. Sa longueur excède assez sensiblement la hauteur maximale du corps.

L'endosquelette de la nageoire anale comprend 10 à 12 ptérygiophores dont les plus antérieurs sont très longs puisque leur extrémité proximale atteint pratiquement les $2 / 3$ de la distance séparant le bord ventral du corps de la partie inférieure des centra vertébraux correspondants. Ils s'intercalent entre les extrémités distales recourbées des hémapophyses correspondantes.

Nageoires pectorales. Les nageoires pectorales se composent d'une quinzaine de rayons dont l'extrémité distale atteint presque la base des rayons des nageoires pelviennes, comme le montre le spécimen MNHN.F.CHD19. On y distingue un long rayon supérieur articulé et non bifurqué, au-dessous duquel on dénombre 14 ou 15 rayons à la fois articulés et bifurqués dont la longueur diminue progressivement vers le bas.

Ceinture scapulaire (Fig. 15). Elle est relativement bien connue car plusieurs de ses composants sont fossilisés à l'état isolé. Le cleithrum est caractérisé par le grand développement de sa branche inférieure qui est longue et relativement étroite (Fig. 16). En revanche, sa branche supérieure, qui s'élargit vers le bas et vers l'arrière, est relativement courte, comme on peut le voir sur le spécimen MNHN.F.CHD10. Langle postéro-ventral de l'os est projeté vers l'arrière. Il émet un petit processus faisant saillie vers le bas.

L'endosquelette de la ceinture scapulaire est fossilisé en connexion avec le cleithrum sur le spécimen MNHN.F.CHD11 (Fig. 17), mais aussi sous forme d'os isolés sur la pièce MNHN.F.CHD2B. Le coracoïde (Co) se termine en pointe vers l'arrière. Au-dessus, la scapula $(\mathrm{Sca})$, de forme générale subtriangulaire est perforé par le foramen scapulaire. Quatre radiaux assurent l'articulation des rayons de la nageoire pectorale. Le radial supérieur est subtriangulaire. Les trois autres radiaux sont subrectangulaires. À la différence des autres, qui entrent en contact avec le bord postérieur de la scapula, le radial inférieur, qui est le plus grand, s'articule avec le coracoïde.

Nageoires pelviennes. Très petites, elles sont formées de six rayons dont l'extrémité distale des plus longs atteint la base du premier rayon de l'anale. Elles sont insérées beaucoup plus près de l'origine de l'anale que de la base des pectorales (la distance pelvo-anale égale $1 / 4$ à $1 / 3$ de la distance pectoro-anale).

Os pelviens. Bien conservés sur le spécimen MNHN.F.CHD12, ils sont relativement massifs puisque leur largeur égale environ les $3 / 4$ de leur longueur (Fig. 18). Ils sont munis d'un processus postéro-interne bien individualisé.

Écailles. De type cyclö̈de, elles sont relativement grandes et, en général, médiocrement conservées. Leur surface est ornée de circuli concentriques. 


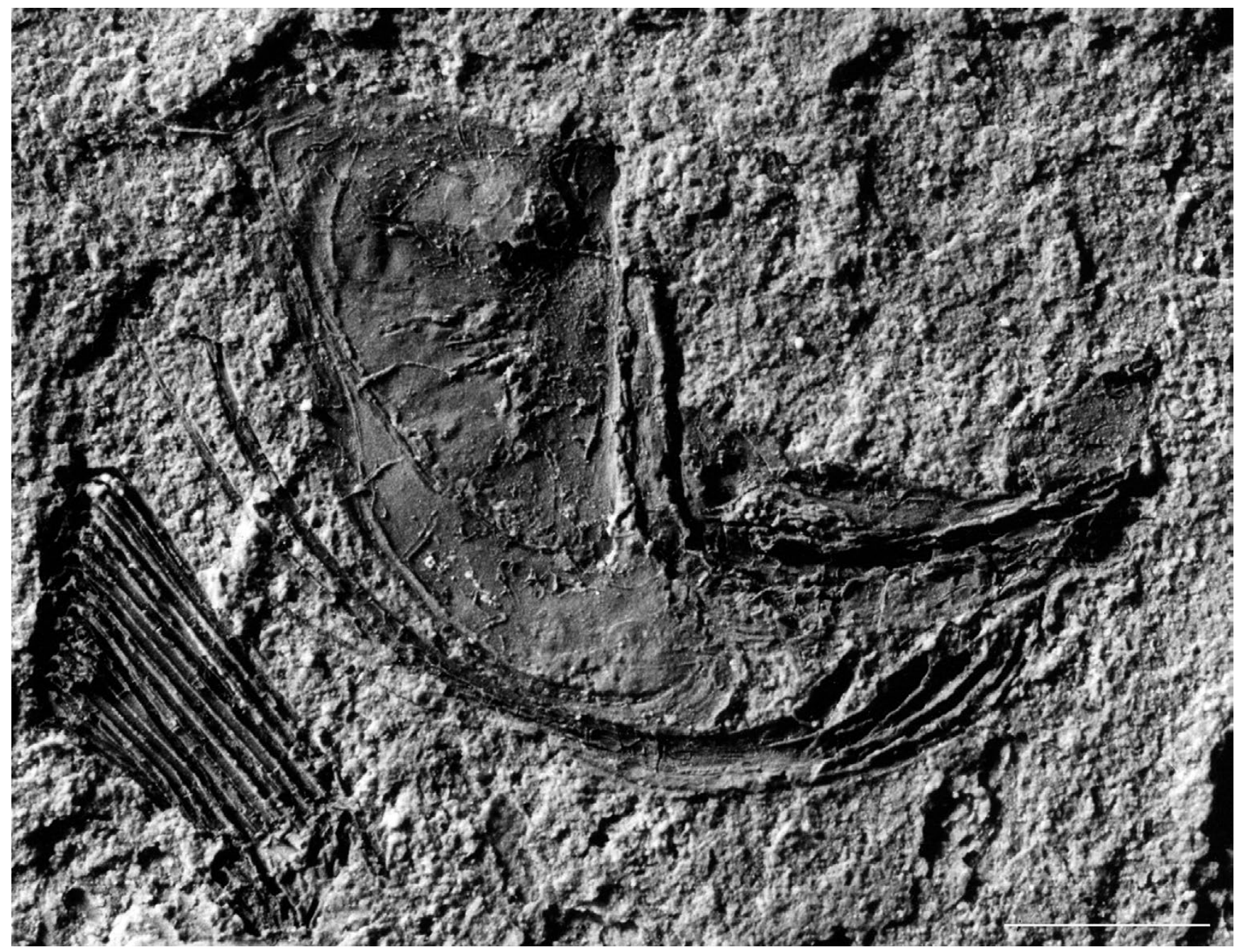

FIG. 13. - Francolebias arvernensis n. sp., os de la région operculaire : spécimen MNHN.F.CHD8G. Échelle: $1 \mathrm{~mm}$.

\section{AFFINITÉS}

Les Cyprinodontiformes fossiles de Chadrat appartiennent à un groupe d'espèces oligocènes qui ont été longtemps attribuées au genre Prolebias jusqu'à ce que Costa (2012) crée pour elles le genre Francolebias. Ces poissons possèdent en effet une série de caractères utilisés dans la diagnose de ce genre:

- prémaxillaire possédant un long processus ascendant ; - nageoire dorsale débutant sensiblement en arrière de l'origine de l'anale;

- nageoire anale dont l'endosquelette comporte des ptérygiophores antérieurs très longs;

- nageoires pelviennes petites, très proches de l'origine de l'anale et portées par des os pelviens massifs;

- squelette caudal axial comportant une plaque hypurale unique et des hémapophyses vertébrales relativement larges.

Au sein du genre Francolebias Costa, les Cyprinodontiformes de Chadrat coexistent avec trois autres espèces dont les caractères méristiques sont relativement homogènes:
- Prolebias rhenanus Gaudant, 1981, du «Plattiger Steinmergel»(Oligocène basal) de Kleinkems (Pays de Bade, Allemagne). Cette espèce est caractérisée à la fois par son squelette caudal axial dont les plaques hypurales inférieure et supérieure sont encore partiellement distinctes, par un nombre un peu plus faible de vertèbres postabdominales et par la forme moins massive de ses os pelviens dont la largeur est inférieure à la moitié de la longueur;

- Prolebias aymardi (Sauvage, 1869), des calcaires marneux de Ronzon (Haute-Loire), d'âge oligocène inférieur (niveau repère mammalien MP 21). Cette espèce se distingue des deux autres par sa nageoire anale dont les ptérygiophores antérieurs sont un peu plus courts que chez les autres espèces; - Prolebias delphinensis Gaudant, 1989, des laminites calcaires de l'Oligocène inférieur de Montbrun-les-Bains (Drôme), se distingue, comme l'a montré Costa (2012: fig. 3D, 3E), par l'épaississement, chez certains individus, des hémapophyses vertébrales situées au-dessus de l'endosquelette de l'anale, alors que d'autres individus possèdent des hémapophyses normales. 


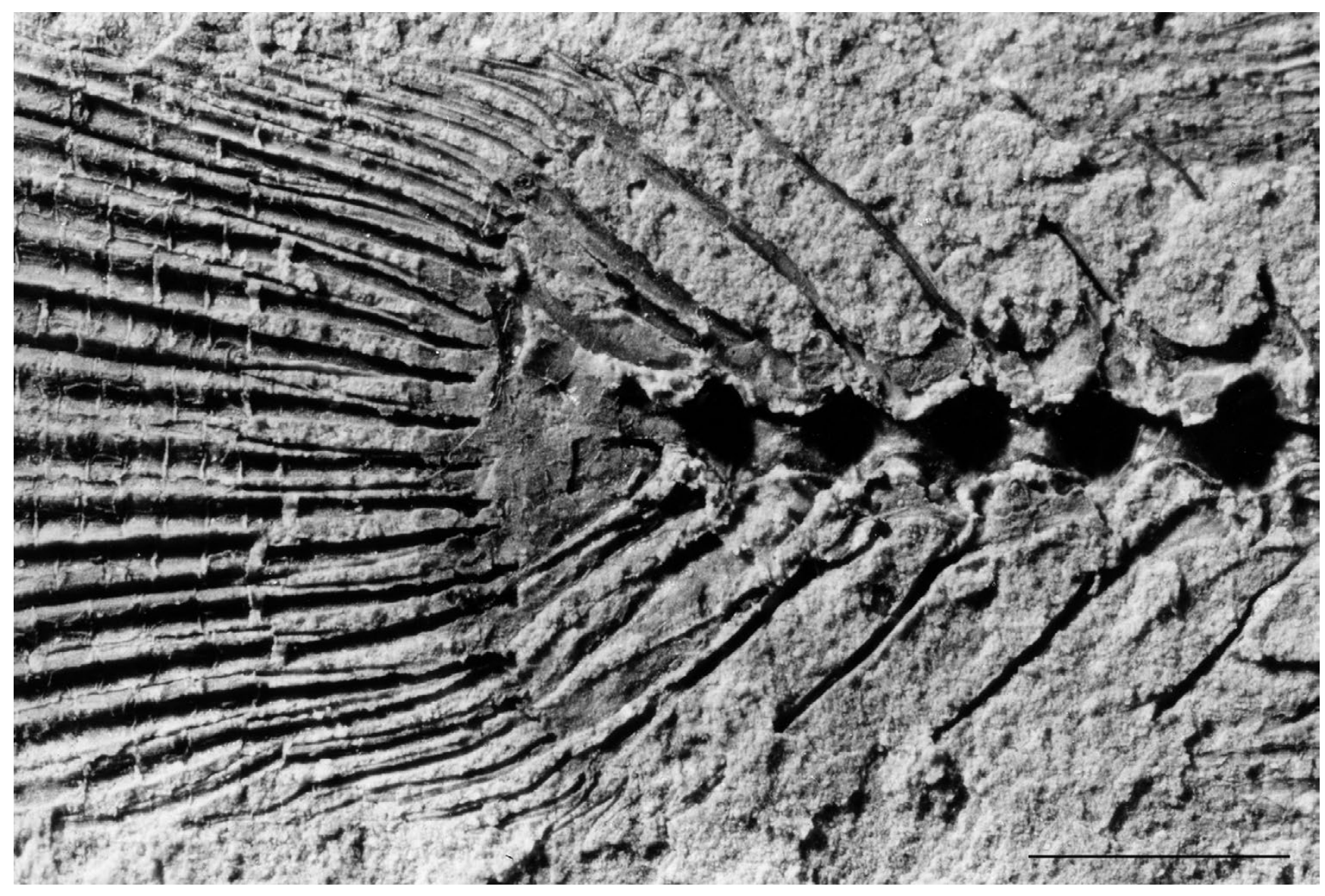

FIG. 14. - A, Francolebias arvernensis n. sp., squelette caudal axial du spécimen MNHN.F.CHD1G. Échelle: 1 mm.

Famille UMBRIDAE

Genre Palaeoesox Voigt, 1934

Palaeoesox cf. weileri (Martini, 1965)

(Fig. 19)

DESCRIPTION ANATOMIQUE

Un unique spécimen très médiocrement fossilisé (MNHN.F.CHD62) a été découvert dans les calcaires finement laminés grisâtres superposés au sapropel fossilifêre. C'est un individu au corps allongé dont la longueur standard mesure $43 \mathrm{~mm}$ et dont la hauteur maximale du corps est comprise environ quatre fois dans cette dimension.

\section{Tête}

Relativement pointue, elle constitue un peu moins du tiers de la longueur du corps. On y observe l'orbite dont le diamètre horizontal est un peu inférieur au tiers de la longueur de la tête. La bouche est relativement grande, la longueur de la mandibule égalant un peu moins de la moitié de la longueur de la tête, son articulation avec le crâne prenant place approximativement sous le milieu de l'orbite. L'opercule est grand: sa largeur égale approximativement un tiers de la longueur de la tête.

Deux rayons branchiostèges acinaciformes.

\section{Colonne vertébrale}

Elle comportait 30 ou 31 centra préuraux $(16 ?+15)$ vertèbres préurales, dont les deux ou trois centra antérieurs sont masqués par l'opercule. On dénombre 15 centra postabdominaux préuraux auxquels font suite deux centra uraux. Les centra préuraux présentent une constriction dans leur région antérieure.

\section{Nageoire caudate}

De la nageoire caudale subsistent seulement les bases des rayons dont il est impossible de déterminer le nombre. Elle est soutenue par un endosquelette dont l'état de conservation est très dégradé.

\section{Nageoire dorsale}

Elle est insérée dans la partie postérieure du corps: la distance antédorsale égale $70 \%$ de la longueur standard. Elle comportait au moins huit rayons. Son endosquelette semble avoir comporté neuf ptérygiophores.

\section{Nageoire anale}

La nageoire anale débute un peu en arrière de la verticale passant par l'origine de la dorsale. La distance antéanale égale $75 \%$ de la longueur standard. Elle semble avoir comporté neuf ou dix rayons que soutenaient huit ou neuf ptérygiophores. 


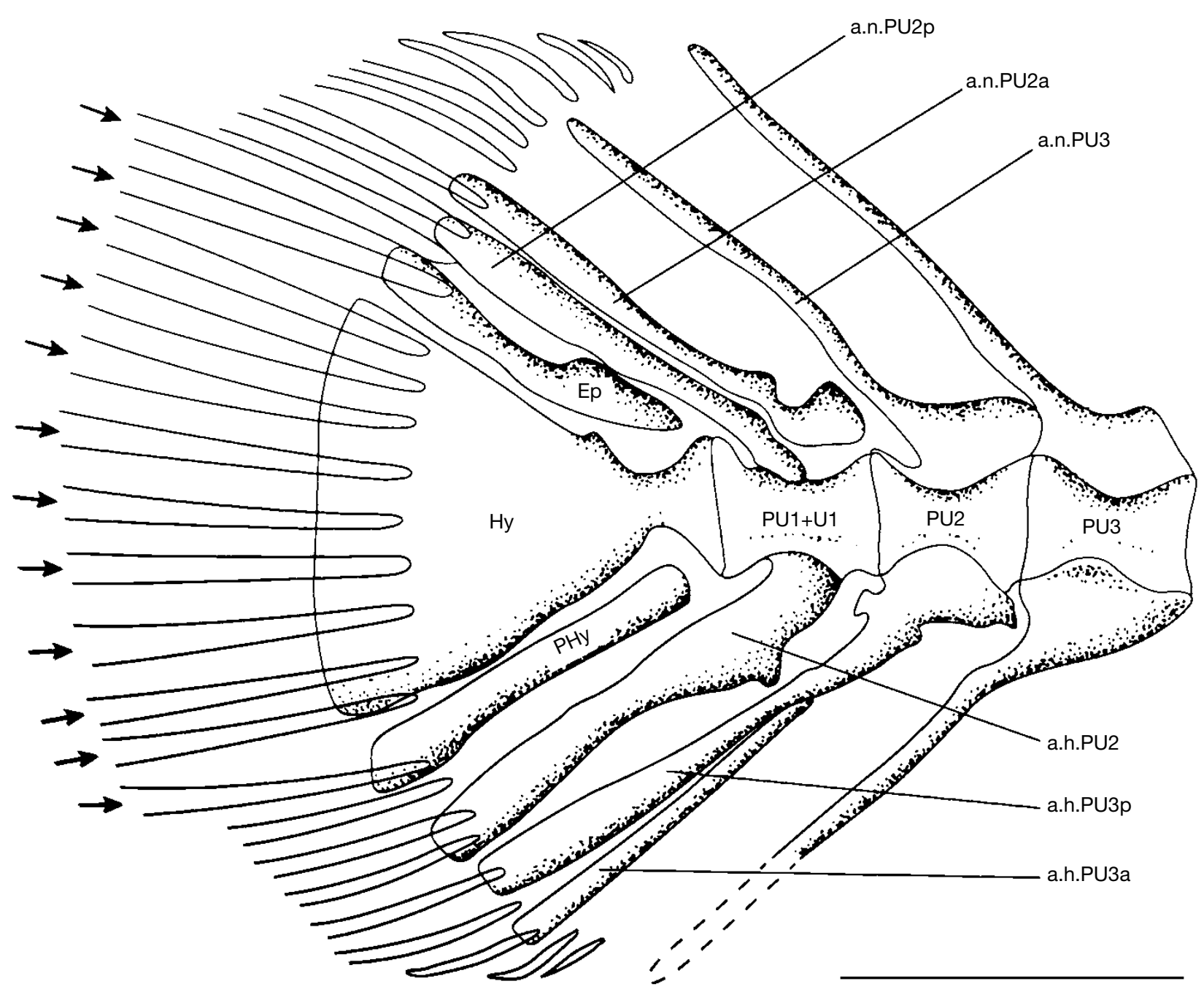

FIG. 14. - B, Francolebias arvernensis n. sp., squelette caudal axial du spécimen MNHN.F.CHD1G. Abréviations: a.h.PU2, hémapophyse portée par le centrum préural libre postérieur; a.h.PU3, hémapophyse dédoublée portée par le centrum préural libre antérieur ; a.n.PU2, neurapophyse dédoublée portée par le centrum préural libre postérieur; a.n.PU3, neurapophyse portée par le centrum préural libre antérieur; Ep, épural ; Hy, plaque hypurale ; PHy, parhypural ; PU1 + U1, complexe uro-terminal ; PU2, centrum préural libre postérieur; PU3, centrum préural libre antérieur. Échelle: $1 \mathrm{~mm}$.

\section{Nageoires pectorales}

Elles sont détruites.

\section{Nageoires pelviennes}

Les nageoires pelviennes, dont on observe les restes de l'os pelvien et quelques débris de rayons, sont insérées approximativement au milieu de l'espace qui devait séparer la base des pectorales de l'origine de l'anale.

\section{Corps}

Le corps était couvert d'écailles qui sont impossibles à décrire en raison de leur mauvais état de conservation.

\section{AFFINITÉS}

En dépit de son état de conservation médiocre, il est possible de rapporter le spécimen de Chadrat à la famille des Umbridae et plus particulièrement au genre Palaeoesox, principalement en raison de la forme générale de son corps, de la position relative des nageoires, de la largeur significative de la région operculaire et de la morphologie des centra vertébraux qui montrent une constriction dans leur partie antérieure. En se référant à notre récente étude des Umbridae fossiles européens connus à ce jour (Gaudant 2012), il apparaît qu'il diffère de l'espèce type du genre Palaeoesox, P. fritzschei Voigt, 1934, de l'Éocène moyen de Geiseltal (Saxe-Anhalt, Allemagne), par son nombre plus faible de vertèbres et par sa nageoire dorsale plus petite, qui comporte deux ou trois ptérygiophores de moins.

Il présente en revanche des affinités étroites avec l'espèce oligocène Palaeoesox weileri, de l'Oligocène inférieur de Sieblos (Hesse, Allemagne), comme le montrent à la fois la composition de sa colonne vertébrale et celle de l'endosquelette de ses nageoires dorsale et anale, ainsi que la forme de son corps. Il diffère en revanche de l'espèce $P$. perpusillus (Agassiz, 1839), du Miocène moyen d'Öhningen (Pays de Bade, Allemagne), qui possède un corps sensiblement plus élancé. C'est pourquoi nous proposons de désigner l'Umbridae fossile de Chadrat comme Palaeoesox cf. weileri. 


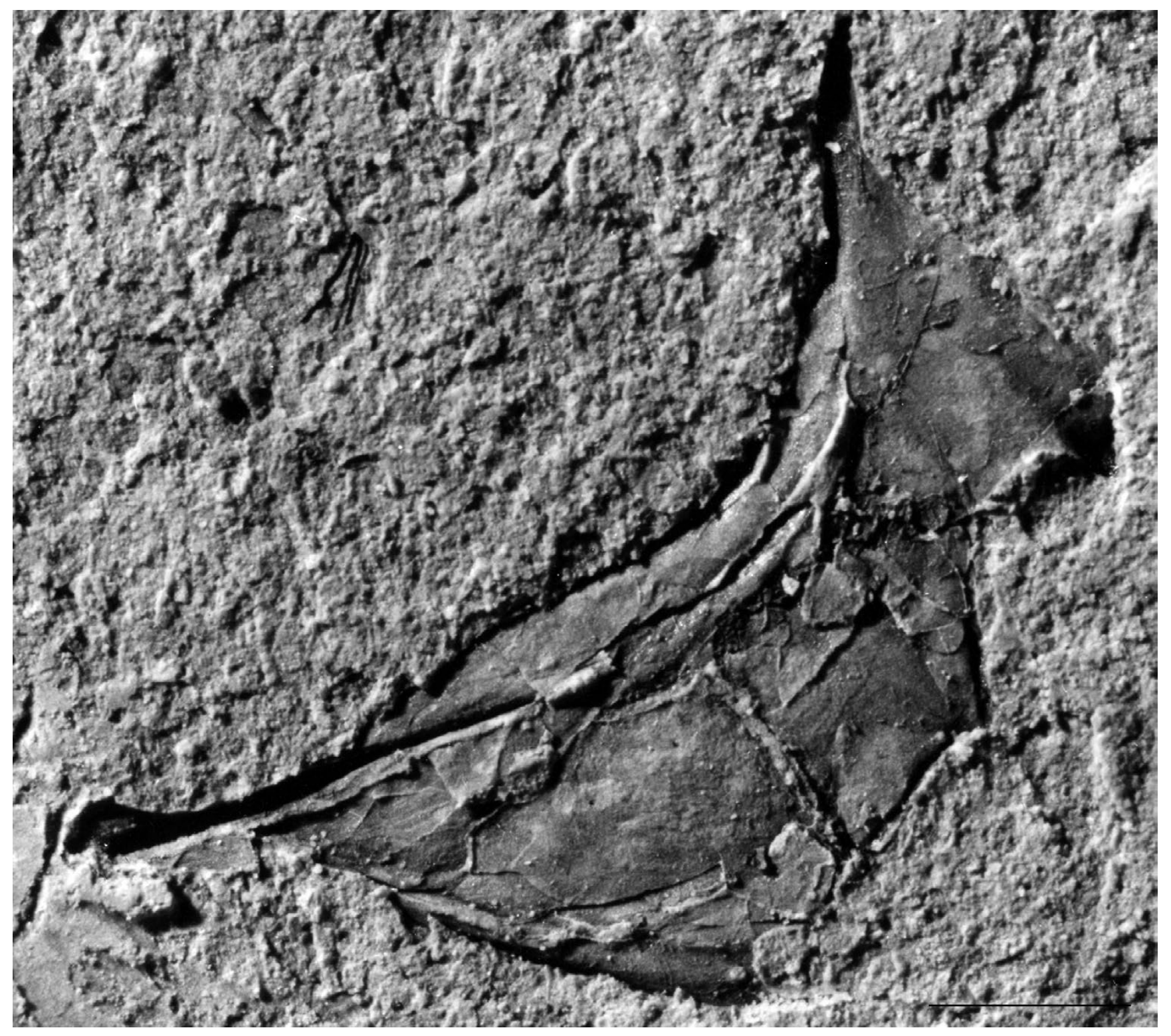

FIG. 15. - Francolebias arvernensis n. sp., ceinture scapulaire. Spécimen MNHN.F.CHD9. Échelle: $1 \mathrm{~mm}$.

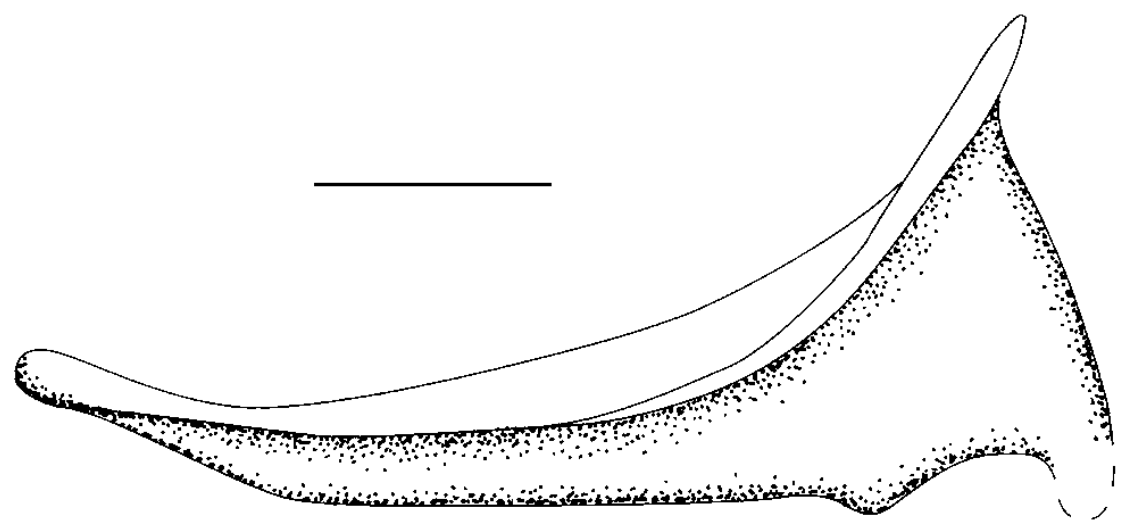

FIG. 16. - Francolebias arvernensis n. sp., cleithrum isolé. Spécimen MNHN.F.CHD10. Échelle: 1 mm. 


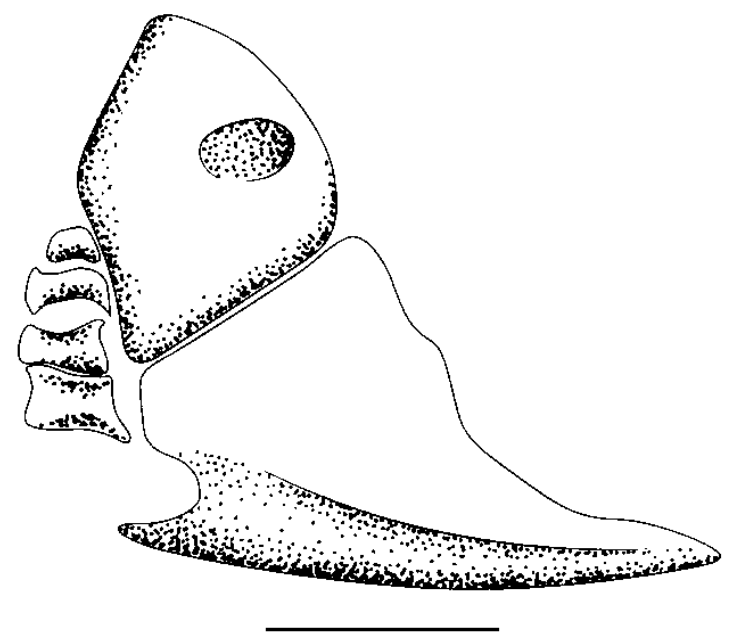

FIG. 17. - Francolebias arvernensis n. sp., endosquelette de la ceinture scapulaire. Spécimen MNHN.F.CHD11. Échelle: 1 mm.

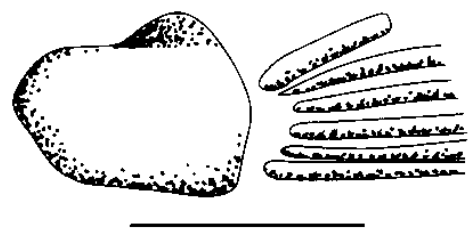

FIG. 18. - Francolebias arvernensis n. sp., os pelvien. Spécimen MNHN.F.CHD12. Échelle: $1 \mathrm{~mm}$.

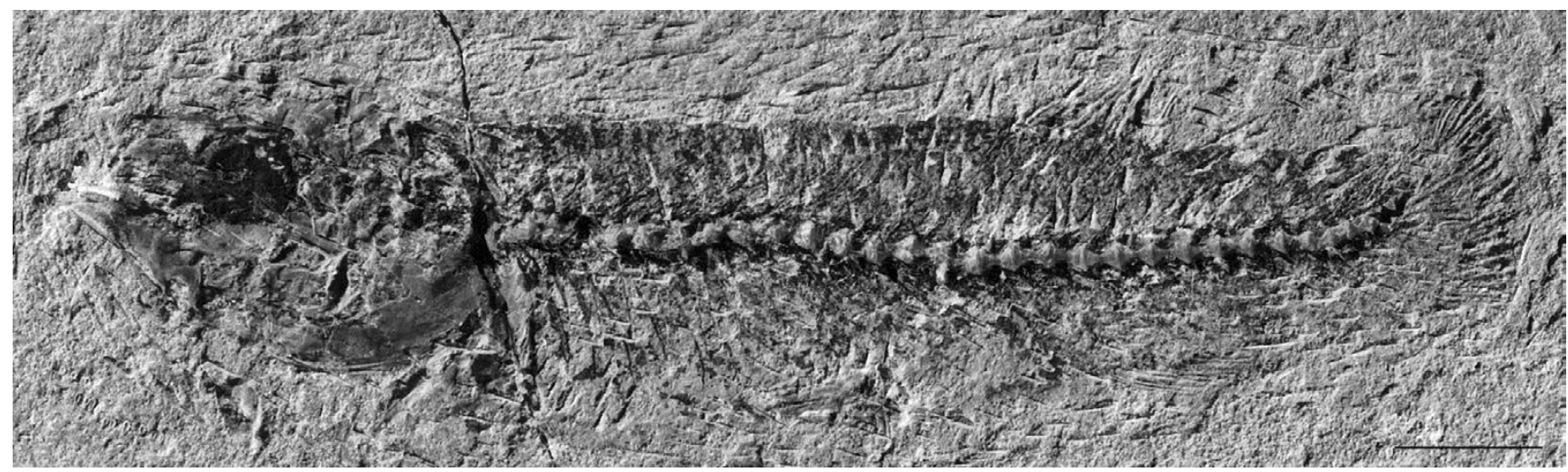

FIG. 19. - Palaeoesox cf. weileri (Martini, 1965), vue générale du spécimen MNHN.F.CHD62. Échelle: 5 mm.

\section{CONCLUSION}

L'identification d'une nouvelle espèce du genre de poissons Cyprinodontiformes Francolebias fournit de précieuses informations à la fois sur l'âge des sapropels fossilifères de Chadrat et des calcaires qui leur sont associés, mais aussi sur leurs conditions de dépôt.

Sur le premier point, il faut savoir que le genre Francolebias n'était connu précédemment que dans trois gisements oligocènes, dont deux sont datés de façon certaine de l'Oligocène basal. En effet, $F$. rhenanus est fossilisé dans une marne en plaquette dont l'âge correspond à celui des «Couches de Pechelbronn moyennes" du Fossé rhénan, qui sont considérées comme l'équivalent des "Glaises à
Cyrènes » qui constituent la base de l'Oligocène aux environs de Paris. D'autre part, F. aymardi, provient du Calcaire de Ronzon, dont la faune mammalienne appartient au niveau repère de Soumailles (MP 21), c'est-à-dire également à l'Oligocène basal.

En l'absence d'informations précises sur l'âge de l'espèce oligocène de Montbrun-les-Bains (Drôme), F. delphinensis, il n'existe donc pas de contre-indication à proposer un âge oligocène basal pour les sapropels fossilifères de Chadrat et les calcaires qui leur sont associés.

D'un point de vue paléoécologique, le mode de vie des Cyprinodontiformes fossiles de Chadrat peut être déduit de la présence de quelques squelettes de Francolebias arvernensis n. sp. associés à un spécimen de Palaeoesox cf. weileri, 
une espèce connue pour avoir fréquenté les eaux douces. Un second argument peut être déduit de la présence de l'espèce $F$. aymardi, qui caractérise le calcaire lacustre de Ronzon (Le Puy-en-Velay, Haute-Loire).

\section{Remerciements}

Philippe Olivier mérite d'être chaleureusement remercié pour sa participation active aux récoltes et pour avoir fait don de nombreux spécimens. C'est également lui qui a mis en évidence la présence de squelettes de poissons, dont celui d'un Umbridae, dans le calcaire gris associé au sapropel. Les rapporteurs, Bettina Reichenbacher et Giorgio Carnevale, sont également remerciés pour les améliorations qu'ils ont permis d'apporter à une première version du manuscrit.

\section{Remerciements de la rédaction}

L'auteur, Jean Gaudant, est décédé durant le processus de publication de son article. La rédaction de Geodiversitas tient donc à remercier deux de ses proches collègues, Giorgio Carnevale, qui a relu la dernière version de l'article avant sa publication et Bettina Reichenbacher, qui a écrit l'hommage à Jean Gaudant inclus dans ce volume (Reichenbacher 2016), et regroupé l'ensemble de sa bibliographie. La rédaction de Geodiversitas souligne, enfin, le plaisir qu'elle a pris à publier les articles de Jean Gaudant, articles dont la pertinence scientifique et la qualité technique n'ont jamais fait défaut.

\section{RÉFÉRENCES}

Costa W. J. E. M. 2012. — Oligocene killifishes (Teleostei, Cyprinodontiformes) from southern France: relationships, taxonomic position, and evidence of internal fertilization. Vertebrate Zoology 62 (3): 371-386.

GAUDANT J. 1981. - Un nouveau Cyprinodontidae (Poisson téléostéen) de l'Oligocène inférieur de Kleinkems (Pays de Bade, Allemagne: Prolebias rhenanus nov. sp. Sciences géologiques, Bulletin 34 (1):3-12.

GAUDANT J. 1988. — Les Cyprinodontiformes (poissons téléostéens) oligocènes de Ronzon, Le Puy-en-Velay (Haute-Loire) : anatomie et signification paléoécologique. Geobios 21 (6): 773-785. http:// dx.doi.org/10.1016/S0016-6995(88)80093-7

GAUDANT J. 1989. - Découverte d'une nouvelle espèce de poissons cyprinodontiformes (Prolebias delphinensis nov. sp.) dans l'Oligocène du bassin de Montbrun-les-Bains (Drôme). Géologie méditerranéenne 16 (4): 355-367.

GAUDANT J. 2012. - An attempt at the palaeontological history of the European mudminnows (Pisces, Teleostei, Umbridae). Neues Jahrbuch für Geologie und Paläontologie Abhandlungen, 263 (2): 93-109. http://dx.doi.org/10.1127/0077-7749/2012/0214

MARTINI E. 1965. — Die Fischfauna von Sieblos/Rhön (Oligozän). 2: Fischreste aus Koproliten. Senckenbergiana lethaea 46a (Weiler Festschrift): 307-314.

PitON L. 1935. - Les poissons fossiles du dussodyle de Chadrat (Puy-de-Dôme). Revue scientifique du Bourbonnais et du Centre de la France (3-4): 37-41.

REICHENBACHER B. 2016. - Jean Gaudant (* 31 December 1939 $\dagger 6$ December 2015) In memoriam. Geodiversitas 38 (3): 327-339. http://dx.doi.org/10.5252/g2016n3a1

SAUVAGE H. E. 1869. - Note sur les poissons du calcaire de Ronzon, près Le Puy-en-Velay. Bulletin de la Société géologique de France, sér. 2, 26: 1069-1075.

VoIGT E. 1934. — Die Fische aus der mitteleozänen Braunkohle des Geiseltales mit besonderer Berücksichtigung der erhaltenen Weichteile. Nova Acta Leopoldina (N.F.) 2 (1/2): 21-146, 14 taf.

Soumis le 5 mai 2015; accepté le 4 janvier 2016; publié le 30 septembre 2016. 
ANNEXE 1. - Diagnoses compilées d'après Piton (1935) pour Haplochilus piacentini et Prolebias stenoura (Sauvage, 1874).

\section{Haplochilus piacentini}

DiAgNOSE COMPILÉE D’APRÈS SA DESCRIPTION PAR PITON (1935). «Longueur $36 \mathrm{~mm}$. Hauteur comprise près de six fois dans la longueur. 32 vertèbres: 12 abdominales et 20 caudales. Pectorales assez grandes; $15-18$ rayons. Pelviennes situées assez près de l'anale; I+5 rayons. Anale longue; 16 rayons. Dorsale assez longue; $10-11$ rayons débutant en arrière de l'anale, aux $3 / 5$ de celle-ci. Caudale comprise 4,5 fois dans la longueur totale; 16 rayons médians et 7-8 rayons marginaux en haut et en bas.»

Un unique exemplaire.

\section{"Prolebias stenoura»}

Diagnose Compilée D’APRÈs Piton (1935). — « Longueur maximale $48 \mathrm{~mm}$. Hauteur maximale du corps comprise 4,5 fois dans la longueur. Tête comprise 4 fois et demie dans la longueur totale. 34 vertèbres: 14 thoraciques et 20 (19) caudales. Pectorales petites: 17 rayons. Dorsale 15 (13-14). Anale débutant à l'aplomb de la dorsale, longue; 15 (14). Caudale comprise 5 fois dans la longueur totale; $19-20$ rayons médians et 5 rayons marginaux dorsaux et ventraux.» 